\title{
On Spaces and Maps of Generalized Inverses
}

\author{
John Z. Hearon* and John W. Evans*
}

(March 26, 1968)

\begin{abstract}
Several classes of generalized inverses of a given $m \times n$ matrix are considered. A collection of continuous maps is given, each of which maps a class of generalized inverses onto a stronger class and the elements of the stronger class are the fixed points of the map. For the case of $E \operatorname{Pr}$ matrices one of these maps is studied in more detail. The various classes of generalized inverses are characterized as subspaces of the space of all $n \times m$ matrices.
\end{abstract}

Key Words: Generalized inverse, linear algebra, matrix.

\section{Introduction}

Several classes of generalized inverses of a given $m \times n$ matrix $A$ are considered.

In section 3 it is shown that the ability to construct a generalized inverse of the weakest class provides the ability to construct a generalized inverse in any one of the stronger classes. Then a collection of continuous maps is given each of which maps a class of generalized inverses onto a stronger class of generalized inverses that remains fixed under the map. In section 4 we characterize these classes of generalized inverses as subspaces of the space of all $n \times m$ matrices and examine in more detail one of the maps given in section 3 .

\section{Preliminaries and Definitions}

We consider only matrices with complex entries. For any matrix $M$ we denote by $\rho(M), R(M)$, $N(M)$ and $M^{*}$ the rank, range, null space and conjugate transpose of $M$. By $I$ we denote an identity matrix the order of which will be clear from the context. By $V^{k}$ we denote a $k$-dimensional vector space over the complex field. If $S_{1}$ and $S_{2}$ are any two sets we denote by $S_{1}-S_{2}$ the set of all elements which are in $S_{1}$ and not in $S_{2}$; by $S_{1} \cup S_{2}$ the union of $S_{1}$ and $S_{2}$; by $S_{1} \cdot S_{2}$ the intersection of $S_{1}$ and $S_{2}$; and by $S_{1} \leqslant S_{2}$ denote that $S_{1}$ is a subset of $S_{2}$. We recall that a homeomorphism is a continuous map which is one to one, onto and has a continuous inverse.

When the matrix $A$ is nonsingular, we denote in the usual way by $A^{-1}$ the inverse of $A$. For generalized inverses we adopt a special terminology as follows: We define five classes of generalized inverses. For a given matrix $A, C_{1}(A)$ is the set of all matrices $B$ such that $A B A=A ; C_{2}(A)$ is the set of all matrices $B \epsilon C_{1}(A)$ such that $B A B=B ; C_{3}(A)$ is the set of all matrices $B \epsilon C_{2}(A)$ such that $A B$ is Hermitian; $C_{3^{\prime}}(A)$ is the set of all matrices such that $B \epsilon C_{2}(A)$ and $B A$ is Hermitian; and $C_{4}(A)$ is the set of all matrices such that $B \epsilon C_{3}(A)$ and $B \epsilon C_{3^{\prime}}(A)$. We call a matrix $B \epsilon C_{i}(A)$, a $C_{i}$-inverse of $A, i=1,2,3,3^{\prime}, 4$. This classification of generalized inverses has been used in previous work to which we will refer $[4,5,6]^{1}$ and related there to other systems of nomenclature which are in use. We note here that the $C_{3^{\prime}}$-inverse is the Goldman-Zelen weak generalized inverse [3] and that $C_{4}(A)=C_{3}(A) \cdot C_{3^{\prime}}(A)$ is a single matrix, the unique Moore-Penrose generalized inverse [10]. There are many statements regarding a $C_{3}$-inverse which of necessity hold for the $C_{3^{\prime}}$-inverse (see [5], other examples occur in sec. 4). But there are contexts in which the role of elements of

\footnotetext{
*An invited paper, present address: Mathematical Research Branch, NIAMD, National Institutes of Health, Bethesda, Md. 20014.

1 Figures in brackets indicate the literature references at the end of this paper.
} 
$C_{3}(A)$ and $C_{3^{\prime}}(A)$ are quite different (see Theorem 7). It is clear that $C_{4}(A) \leqslant C_{3}(A) \leqslant C_{2}(A) \leqslant C_{1}(A)$ and that $C_{4}(A) \leqslant C_{3^{\prime}}(A) \leqslant C_{2}(A) \leqslant C_{1}(A)$. We define four classes of strict generalized inverses as follows: A strict $C_{1}$-inverse of $A$ is any matrix $B \epsilon\left(C_{1}(A)-C_{2}(A)\right)$; a strict $C_{2}$-inverse of $A$ is any matrix $B \boldsymbol{\epsilon}\left(C_{2}(A)-C_{3}(A) \cup C_{3^{\prime}}(A)\right)$; a strict $C_{3^{-}}$-inverse of $A$ is any matrix $B \epsilon\left(C_{3}(A)-C_{4}(A)\right)$; a strict $C_{3^{\prime}}$-inverse of $A$ is any matrix $B \epsilon\left(C_{3^{\prime}}(A)-C_{4}(A)\right)$. We will sometimes say $B \epsilon C_{i}(A)$ and is strict, meaning that $B$ is a strict $C_{i}$-inverse of $A$, and when it is clear from the context that $B$ is in a given class we will simply say that $B$ is strict when it is a strict member of that class. Consideration of strictness leads to alternative characterizations of the classes of generalized inverses defined above and studies of this type were first carried out by Rohde [11, 12]. For example, Lemma 1 below, gives necessary and sufficient conditions for $B \epsilon C_{1}(A)$ to be strict. If certain demands are made on $A$, and especially if demands are made on $A$ and a generalized inverse of $A$, there may exist no strict generalized inverses in a certain class. Such cases are known [5] and others appear in section 4 .

Finally we recall that a matrix $A$ is called an $E P$ matrix if $N(A)=N\left(A^{*}\right)$, in particular if $A$ is $E P$ and $\rho(A)=r$ we say that $A$ is an $E P r$ matrix [8], or merely that $A$ is $E P r$. For ready reference we record the following known lemmas (one dealing with $E P$ matrices) to which repeated reference will be made.

LEMMA 1. The matrix $\mathrm{B}$ is a $\mathrm{C}_{1}$-inverse of $\mathrm{A}$ if and only if $\mathrm{BA}$ is a projection and $\rho(\mathrm{A})=\rho(\mathrm{BA})$; and if and only if $\mathrm{AB}$ is a projection and $\rho(\mathrm{A})=\rho(\mathrm{AB})$. If $\mathrm{B} \epsilon \mathrm{C}_{1}(\mathrm{~A})$, then $\rho(\mathrm{B}) \geqslant \rho(\mathrm{A})$ with strict equality if and only if $\mathrm{B} \epsilon \mathrm{C}_{2}(\mathrm{~A})$.

The first statement of Lemma 1 is Corollary 1 of [4]. The second statement combines Lemmas 1 and 2 of [4], both proved in a different way by Rohde [12].

Lemma 2. Let $\mathrm{P}$ be an $\mathrm{n} \times \mathrm{m}$ matrix, $\mathrm{Q}$ and $\mathrm{R}$ be $\mathrm{m} \times \mathrm{n}$ matrices. If $\mathrm{PQ}$ is a projection such that $\rho(\mathrm{PQ})=\rho(\mathrm{Q})$ and $\mathrm{N}(\mathrm{R})=\mathrm{N}(\mathrm{Q})$, then $\mathrm{RPQ}=\mathrm{R}$.

LEMMA 3. $\mathrm{P}^{*}=\mathrm{P}^{*} \mathrm{QP}$ if and only if $\mathrm{Q} \epsilon \mathrm{C}_{1}(\mathrm{P})$ and $\mathrm{N}(\mathrm{P})=\mathrm{N}\left(\mathrm{P}^{*}\right)$. Further, $\mathrm{P}^{*}=\mathrm{P}^{*} \mathrm{QP}$ if and only if $\mathrm{P}^{*}=\mathrm{PQP}^{*}$.

Lemma 2 is Lemma 3 of [4] and Lemma 3 is Corollary 2 of [4].

\section{Maps and Constructions}

We take for granted the existence of, and known methods for constructing, a $C_{1}$-inverse of an arbitrary matrix ${ }^{2}[1,11]$. The first theorem shows that the ability to construct a $C_{1}$-inverse of an arbitrary matrix gives us the ability to construct a matrix in any given class of the five classes of generalized inverses which we have defined.

Theorem 1. Let $\mathrm{A}$ be a given matrix. Define $\mathrm{H}=\mathrm{A}^{*} \mathrm{~A}, \mathrm{~J}=\mathrm{AA}^{*}$ and let $\mathrm{B}_{1} \in \mathrm{C}_{1}(\mathrm{~A}), \mathrm{K} \epsilon \mathrm{C}_{1}(\mathrm{H})$,

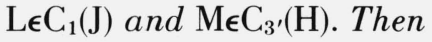

(i) $\mathrm{B}_{2}=\mathrm{B}_{1} \mathrm{AB}_{1}$ is in $\mathrm{C}_{2}(\mathrm{~A})$ and every matrix in $\mathrm{C}_{2}(\mathrm{~A})$ can be so expressed for some $\mathrm{B}_{1} \in \mathrm{C}_{1}(\mathrm{~A})$.

(ii) $\mathrm{B}_{3}=\mathrm{KA}^{*}$ is in $\mathrm{C}_{3}(\mathrm{~A})$ and every matrix in $\mathrm{C}_{3}(\mathrm{~A})$ can be so expressed for some $\mathrm{K}_{\epsilon} \mathrm{C}_{1}(\mathrm{H})$.

(iii) $\mathrm{B}_{3^{\prime}}=\mathrm{A}^{*} \mathrm{~L}$ is in $\mathrm{C}_{3^{\prime}}(\mathrm{A})$ and every matrix in $\mathrm{C}_{3^{\prime}}(\mathrm{A})$ can be so expressed for some $\mathrm{L}_{\epsilon} \mathrm{C}_{1}(\mathrm{~J})$.

(iv) $\mathrm{B}_{4}=\mathrm{MA}^{*}$ is the $\mathrm{C}_{4}$-inverse of $\mathrm{A}$.

(v) Let $\rho(\mathrm{A})=\rho\left(\mathrm{A}^{2}\right)$. Then $\mathrm{B}=\mathrm{AWA}$ is in $\mathrm{C}_{2}(\mathrm{~A})$ and commutes with $\mathrm{A}$ if and only if $\mathrm{W} \epsilon \mathrm{C}_{1}\left(\mathrm{~A}^{3}\right)$.

Proof. (i): That $B_{2}=B_{1} A B_{1}$ is in $C_{2}(A)$ is a special case of a known theorem [4]. That every matrix in $C_{2}(A)$ can be so expressed is obvious. (ii): If $B_{3}=K A^{*}$ we have $B_{3} A=K H$. Then by Lemma 1, $B_{3} A$ is a projection, $\rho\left(B_{3} A\right)=\rho(H)=\rho(A)$ and thus $B_{3} \in C_{1}(A)$. By Lemma $1, \rho\left(B_{3}\right) \geqslant \rho(A)$ but also $\rho\left(B_{3}\right)=\rho\left(K A^{*}\right) \leqslant \rho(A)$. Hence $\rho\left(B_{3}\right)=\rho(A)$ and, by Lemma $1, B_{3} \in C_{2}(A)$. By Lemma 1 , $A B_{3}$ is a projection with rank $\rho(A)$ and from $A B_{3}=A K A^{*}$ it is clear that $N\left(A B_{3}\right)=N\left(\left(A B_{3}\right)^{*}\right)$ and $A B_{3}$ is Hermitian. Thus $B_{3} \epsilon C_{3}(A)$. Conversely if $B_{3} \epsilon C_{3}(A)$ we have $B_{3}=B_{3} A B_{3}=B_{3} B_{3}^{*} A^{*}$ and we have to show that $B_{3} B_{3}^{*} \in C_{1}(H)$. But by Lemma $1, B_{3} A$ is a projection with rank $\rho(A)=\rho(H)$, and $B_{3} A=B_{3} B_{3}^{*} H$ shows, by Lemma 1, that $B_{3} B_{3}^{*} \in C_{1}(H)$. (iii): The proof of (iii) parallels that of (ii) in an obvious manner. (iv): Let $B_{4}=M A^{*}$. Then by (ii) we have $B_{4} \epsilon C_{3}(A)$. We now observe that $B_{4} A=M H$ is Hermitian and hence $B_{4} \epsilon C_{4}(A)$. We note that conversely if $B_{4} \epsilon C_{4}(A)$ we have $B_{4}=B_{4} B_{4}^{*} A^{*}$, and $B_{4} B_{4}^{*} \epsilon C_{4}(H)$ [10]. (v): If $W \epsilon C_{1}\left(A^{3}\right)$, then by Lemma $1, W A^{3}=\left(W A^{2}\right) A$ is a projec-

\footnotetext{
${ }^{2}$ See also (i) of Theorem 4.
} 
tion with rank $\rho\left(A^{3}\right)=\rho(A)$ and $W A^{2} \epsilon C_{1}(A)$. This being the case we have, by Lemma $1, A W A^{2}=B A$ is a projection of rank $\rho(A)$ and $B \epsilon C_{1}(A)$. But $\rho(B) \leqslant \rho(A)$ and hence, by Lemma $1, B \epsilon C_{2}(A)$. The projections $A B=A^{2} W A$ and $B A=A W A^{2}$ clearly both have null space $N(A)$ and range $R(A)$. Thus $A B=B A$. Now assume $B \epsilon C_{2}(A)$ and $A B=B A$. Then $A^{3} W A^{3}=A^{2} B A^{2}=A^{3}$ shows that $W \epsilon C_{1}\left(A^{3}\right)$. This completes the proof of the theorem.

REMARK: Goldman and Zelen [3] have proved that $B_{3^{\prime}}=A^{*} N$ is in $C_{3^{\prime}}(A)$ if $N \epsilon C_{2}(J)$, and that every matrix in $C_{3^{\prime}}(A)$ can be so expressed for some $N \epsilon C_{2}(J)$. The above proof of (iii) shows that this theorem goes through under the weaker condition $N \epsilon C_{1}(J)$. However we note that if $B \epsilon C_{3}(A)$, then $B B^{*}$ is in fact in $C_{2}(H)$. For, in the proof of (ii) we have shown $B B^{*} \epsilon C_{1}(H)$, and from Lemma 1 and $\rho\left(B B^{*}\right)=\rho(B)=\rho(A)=\rho(H)$, we have $B B^{*} \epsilon C_{2}(H)$. By the same kind of argument $B^{*} B \epsilon C_{2}(J)$ whenever $B \epsilon C_{3^{\prime}}(A)$.

It is well known that for any matrix $A$, the $C_{4}$-inverse of $A$ exists and is unique. The next theorem gives a set of mappings which map an arbitrary $C_{1}$-inverse onto a stronger class of inverse and fixes the stronger class of inverse. These maps are continuous and have differentiability properties of which considerable use is made in a subsequent paper [7].

We first give two lemmas which deal with the difference of two $C_{1}$-inverses.

LeMmA 4. If $\mathrm{B}_{1}$ and $\mathrm{B}_{2}$ are in $\mathrm{C}_{1}(\mathrm{~A})$ then $\mathrm{A}\left(\mathrm{B}_{1}-\mathrm{B}_{2}\right) \mathrm{A}=0$. Conversely, if $\mathrm{ADA}=0$, then for any $\mathrm{B}_{1} \epsilon \mathrm{C}_{1}(\mathrm{~A})$ we have $\left(\mathrm{B}_{1}+\mathrm{D}\right) \epsilon \mathrm{C}_{1}(\mathrm{~A})$.

Proof. The proof is obvious from the definition of $C_{1}(A)$.

LeMma 5. If $\mathrm{ADA}=0$ then $\mathrm{D}=\mathrm{P}_{\mathrm{N}} \mathrm{DP} \mathrm{P}_{\mathrm{R}}+\mathrm{D}\left(\mathrm{I}-\mathrm{P}_{\mathrm{R}}\right)$, where $\mathrm{P}_{\mathrm{N}}$ and $\mathrm{P}_{\mathrm{R}}$ are any projections onto $\mathrm{N}(\mathrm{A})$ and $\mathrm{R}(\mathrm{A})$, respectively. Conversely, for any $\mathrm{D}$ of the form $\mathrm{D}=\mathrm{P}_{\mathrm{N}} \mathrm{Z}_{1}+\mathrm{Z}_{2}\left(\mathrm{I}-\mathrm{P}_{\mathrm{R}}\right)$, where $\mathrm{Z}_{1}$ and $\mathrm{Z}_{2}$ are arbitrary matrices such that the indicated products exist, we have $\mathrm{ADA}=0$.

Proof. If $A D A=0$, then $P_{N} D P_{R}=D P_{R}$. We now have $D=D P_{R}+D\left(I-P_{R}\right)=P_{N} D P_{R}+D\left(I-P_{R}\right)$ and the first statement is proved. The converse is obvious since $\left(I-P_{R}\right) A=0$ and each column of $P_{N} Z_{1}$ is in $N(A)$.

Theorem 2. Let $\mathrm{A}$ be an $\mathrm{m} \times \mathrm{n}$ matrix, $\mathrm{B}^{+}$the $\mathrm{C}_{4}$-inverse of $\mathrm{A}, \mathrm{P}_{\mathrm{N}}$ and $\mathrm{P}_{R}$ any projections onto $\mathrm{N}(\mathrm{A})$ and $\mathrm{R}(\mathrm{A})$, respectively, and $\mathrm{B}$ an $\mathrm{n} \times \mathrm{m}$ matrix. Then

(i) $\varphi_{1}(\mathrm{~B})=\mathrm{B}^{+}+\mathrm{P}_{\mathrm{N}}\left(\mathrm{B}-\mathrm{B}^{+}\right) \mathrm{P}_{\mathrm{R}}+\left(\mathrm{B}-\mathrm{B}^{+}\right)\left(\mathrm{I}-\mathrm{P}_{\mathrm{R}}\right)$ is in $\mathrm{C}_{1}(\mathrm{~A})$ and $\varphi_{1}(\mathrm{~B})=\mathrm{B}$ if and only if $\mathrm{B} \epsilon \mathrm{C}_{1}(\mathrm{~A})$.

(ii) $\varphi_{2}(\mathrm{~B})=\mathrm{BAB}$ is in $\mathrm{C}_{2}(\mathrm{~A})$ whenever $\mathrm{B} \epsilon \mathrm{C}_{1}(\mathrm{~A})$. In this case, $\varphi_{2}(\mathrm{~B})=\mathrm{B}$ if and only if $\mathrm{B} \epsilon \mathrm{C}_{2}(\mathrm{~A})$.

(iii) $\varphi_{3}(\mathrm{~B})=\mathrm{BAB}^{+}$is in $\mathrm{C}_{3}(\mathrm{~A})$ if and only if $\mathrm{B} \epsilon \mathrm{C}_{1}(\mathrm{~A})$. In this case, $\varphi_{3}(\mathrm{~B})=\mathrm{B}$ if and only if $\mathrm{B} \epsilon \mathrm{C}_{3}(\mathrm{~A})$.

(iv) $\varphi_{3^{\prime}}(\mathrm{B})=\mathrm{B}^{+} \mathrm{AB}$ is in $\mathrm{C}_{3^{\prime}}(\mathrm{A})$ if and only if $\mathrm{B} \epsilon \mathrm{C}_{1}(\mathrm{~A})$. In this case, $\varphi_{3^{\prime}}(\mathrm{B})=\mathrm{B}$ if and only if

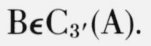

PROOF.

(i) Let $C=\varphi_{1}(B)-B^{+}$. Then by the second part of Lemma $5, A C A=0$ and, by Lemma 4, $\varphi_{1}(B)=B^{+}+C$ is in $C_{1}(A)$. If $B \epsilon C_{1}(A)$, then $A\left(B-B^{+}\right) A=0$ and, by the first part of Lemma 5 , we have $C=B-B^{+}$, but then $B^{+}+C=\varphi_{1}(B)=B$. Conversely if $\varphi_{1}(B)=B$ then $B \in C_{1}(A)$.

(ii) If $B \epsilon C_{1}(A)$, that $\varphi_{2}(B) \epsilon C_{2}(A)$ is a special case of a known theorem [4]. If $B \epsilon C_{1}(A)$ then $\varphi_{2}(B)=B$ if and only if $B \epsilon C_{2}(A)$ follows from the definition of $C_{2}(A)$.

(iii) If $B \epsilon C_{1}(A)$, by a known theorem [4] we have $\varphi_{3}(B) \epsilon C_{2}(A)$. But $A \varphi_{3}(B)=A B^{+}$shows that $A \varphi_{3}(B)$ is Hermitian and hence $\varphi_{3}(B) \epsilon C_{3}(A)$. Conversely, if $\varphi_{3}(B) \epsilon C_{3}(A)$, then $\varphi_{3}(B) A=B A$ is a projection with rank $\rho(A)$, and by Lemma $1, B \epsilon C_{1}(A)$. If $B \epsilon C_{3}(A)$ then the projections $A B$ and $A B^{+}$are Hermitian and we have $N(B)=N\left(B^{+}\right)=N\left(A^{*}\right)$. From Lemma 2 it follows that $B=B A B^{+}=\varphi_{3}(B)$.

(iv) The proof of (iv) is an obvious parallel of that of (iii).

It is known that every square matrix of rank $r$ has $C_{2}$-inverses which are $\operatorname{EPr}$ [4]. When $A$ is $E P r$ the construction of $C_{2}$-inverses which are $E P r$ is particularly simple.

Theorem 3. Let $\mathrm{A}$ be EPr. Then $\psi(\mathrm{B})=\mathrm{BA}^{*} \mathrm{~B}^{*}$ is in $\mathrm{C}_{2}(\mathrm{~A})$ and is $\mathrm{EPr}$ whenever $\mathrm{B} \epsilon \mathrm{C}_{2}(\mathrm{~A})$. In that case $\psi(\mathrm{B})=\mathrm{B}$ if and only if $\mathrm{B}$ is $\mathrm{EPr}$.

Proof. That $\psi(B)$ is in $C_{2}(A)$ and is $E P r$ when $B$ is an arbitrary matrix in $C_{2}(A)$ is Lemma 5 of [4]. If $B$ is $E P r$, then, since $B \epsilon C_{2}(A)$ implies $A \epsilon C_{2}(B)$, we can apply Lemma 3 to write $B=B A^{*} B^{*}$ $=\psi(B)$. 


\section{Subspaces of Generalized Inverses}

In this section we will characterize the classes of generalized inverses previously discussed as subspaces of the space of all $n \times m$ matrices with complex entries viewed as a vector space $V^{n m}$ over the complex numbers.

THEOREM 4. Let A be an $\mathrm{m} \times \mathrm{n}$ matrix of rank $\mathrm{r}$. Then

(i) The collection of all $\mathrm{C}_{1}$-inverses of $\mathrm{A}$ is an affine space in $\mathrm{V}^{\mathrm{nm}}$ of dimension $\mathrm{nm}-\mathrm{r}^{2}$.

(ii) The collection of all $\mathrm{C}_{2}$-inverses of $\mathrm{A}$ is an algebraic variety in $\mathrm{V}^{\mathrm{nm}}$ which is homeomorphic to a linear space of dimension $\left(n m-r^{2}\right)-(m-r)(n-r)$.

(iii) The collection of all $\mathrm{C}_{3}$-inverses of $\mathrm{A}$ is an affine space of $\mathrm{V}^{\mathrm{nm}}$ of dimension $\left(\mathrm{nm}-\mathrm{r}^{2}\right)-(\mathrm{n}-\mathrm{r})$ $\mathrm{r}-(\mathrm{n}-\mathrm{r})(\mathrm{m}-\mathrm{r})=\mathrm{r}(\mathrm{m}-\mathrm{r})$.

(iv) The collection of all $\mathrm{C}_{3^{\prime}}$-inverses of $\mathrm{A}$ is an affine space of $\mathrm{V}^{\mathrm{nm}}$ of dimension $\mathrm{r}(\mathrm{n}-\mathrm{r})$.

Proof: Any matrix $A$ can be written as $Q M R$ where $Q$ and $R$ are unitary and $M=\left(\begin{array}{rr}D & 0 \\ 0 & 0\end{array}\right)$ with $D$ diagonal and nonsingular and with $\rho(D)=\rho(A)$ [2]. It is easily seen that $B \epsilon C_{i}(A)$ if and only if $R B Q \epsilon C_{i}(M), i=1,2,3,3^{\prime}, 4$. Let $R B Q=\left(\begin{array}{ll}U & V \\ W & X\end{array}\right)$. Then the following may be derived directly from the definitions of the types of generalized inverses considered.

(i) $B \in C_{1}(A)$ if and only if $U=D^{-1}$.

(ii) $B \epsilon C_{2}(A)$ if and only if $B \epsilon C_{1}(A)$ and $X=W D V$.

(iii) $B \epsilon C_{3}(A)$ if and only if $B \epsilon C_{2}(A)$ and $V=0$.

(iv) $B \epsilon C_{3^{\prime}}(A)$ if and only if $B \epsilon C_{2}(A)$ and $W=0$.

The dimension and nature of the subspaces given in the theorem then follows from the dimension of the matrices $V, W$, and $X$ not fixed by the requirement that $B$ belong to a particular class of generalized inverses, and from the nature of the stated relations among them.

In case $A$ is $n \times n$ and is $E P r$ we have the following theorem.

Theorem 5. Let $\mathrm{A}$ be $\mathrm{n} \times \mathrm{n}$ and EPr. Then the EPr matrices of $\mathrm{C}_{2}(\mathrm{~A})$ form an algebraic variety of $\mathrm{V}^{\mathrm{n}^{2}}$ which is homeomorphic to a linear space of dimension $\mathrm{r}(\mathrm{n}-\mathrm{r})$.

Proof. Since $A$ is $E P r$ it is known [8] that there exists a unitary matrix $Q$ such that

$$
A=Q\left(\begin{array}{ll}
A_{1} & 0 \\
0 & 0
\end{array}\right) Q^{-1}
$$

where $A_{1}$ is $r \times r$ and nonsingular. It is easily seen that $B \epsilon C_{2}(A)$ if and only if

$$
Q^{-1} B Q=\left(\begin{array}{ll}
A_{1}^{-1} & U \\
V & V A_{1} U
\end{array}\right)
$$

where $U$ and $V$ are arbitrary. By applying the map $\psi$ of Theorem 3 we see that $B \epsilon C_{2}(A)$ is $E P r$ if and only if

$$
Q^{-1} B Q=\left(\begin{array}{cc}
A_{1}^{-1} & A_{1}^{-1} A_{1}^{*} V^{*} \\
V & V A_{1}^{*} V^{*}
\end{array}\right) .
$$

The theorem follows immediately from equating these two expressions.

When $A$ is $E P r$ the question of strictness of generalized inverses of $A$ becomes rather special. The following lemma shows that when $A$ is $E P r$, there exists no strict $C_{2}$-inverse which commutes with $A$, no strict $C_{3}$-inverse and no strict $C_{3^{\prime}}$-inverse.

LEMma 6. Let A be EPr. Then the intersection of the set of all $\mathrm{EPr}_{2}$-inverses of $\mathrm{A}$ with $\mathrm{C}_{3}(\mathrm{~A})$ (or with $\mathrm{C}_{3^{\prime}}(\mathrm{A})$ ) is the $\mathrm{C}_{4}$-inverse of $\mathrm{A}$ which in this case is the unique $\mathrm{C}_{2}$-inverse of $\mathrm{A}$ which commutes with A. 
Proof. If $B \epsilon C_{3}(A)$ and is $E \operatorname{Pr}$ then we have that $A B$ is Hermitian and $N(A B)=N(B)=N(A)$. Also $N(B A)=N(A)$ and $N\left((B A)^{*}\right)=N(B)$. Thus $N(B A)=N\left((B A)^{*}\right), B A$ is Hermitian and $B \epsilon C_{4}(A)$. An exactly parallel proof shows that if $B \epsilon C_{3^{\prime}}(A)$ and is $E \operatorname{Pr}$ then $B \epsilon C_{4}(A)$. It is known that for any $G$ such that $\rho(G)=\rho\left(G^{2}\right)$ there exists a $C_{2}$-inverse which commutes with $G$ and this matrix is uniquely determined by $G$ [5]. If $A$ is $E P r$, then $\rho(A)=\rho\left(A^{2}\right)$ [8], and there is a unique $B \epsilon C_{2}(A)$ which commutes with $A$. By a known theorem [9], a matrix commutes with its $C_{4}$-inverse if and only if the matrix is $E P r$; and, by this very theorem, the $C_{4}$-inverse of an $E P r$ matrix is itself $E P r$. Thus, since the $C_{4}$-inverse of $A$ is in $C_{2}(A)$ and commutes with $A$, it is the unique $B \epsilon C_{2}(A)$ which commutes with $A$ and that unique $B \epsilon C_{2}(A)$ must be $E \operatorname{Pr}$. This completes the proof of the lemma.

REMARK. Lemma 6 shows that in Lemma 2 of [5] the condition of normality can be weakened to $E P r$.

We now use Lemma 6 to study the strictness of the $E \operatorname{Pr} C_{2}$-inverses of an $E \operatorname{Pr}$ matrix as established by the following theorem.

Theorem 7. Let $\mathrm{A}$ be $\mathrm{EPr}$ and let $\psi$ be the map of Theorem 3. Then $\psi$ sends all of $\mathrm{C}_{3^{\prime}}(\mathrm{A})$ to $\mathrm{C}_{4}(\mathrm{~A})$ and the remainder of $\mathrm{C}_{2}(\mathrm{~A})$ into the set of all strict $\mathrm{C}_{2}$-inverses of $\mathrm{A}$ which are $\mathrm{EPr}$.

Proof. From Lemma 3 we have

$$
\begin{aligned}
& A \psi(B)=A B A^{*} B^{*}=A^{*} B^{*} \\
& \psi(B) A=B A^{*} B^{*} A=B A .
\end{aligned}
$$

Since $\psi(B) \epsilon C_{2}(A)$ and is $E P r$, by Theorem 3, we have by Lemma 6 that if $\psi(B)$ is not strict, then $\psi(B) \epsilon C_{4}(A)$. It follows from the display that if $\psi(B)$ is not strict then $B \epsilon C_{3^{\prime}}(A)$. Conversely if $B \epsilon C_{3^{\prime}}(A)$ then the display shows that $\psi(B) \epsilon C_{4}(A)$. Thus $\psi$ sends all of $C_{3^{\prime}}(A)$ and only elements in $C_{3^{\prime}}(A)$ to $C_{4}(A)$. The remainder of $C_{2}(A)$ are those elements in $C_{2}(A)$ which are strict and those in $C_{3}(A)$ which are strict. We now show that $\psi$ sends these elements into strict $C_{2}$-inverses. If $B \epsilon C_{3}(A)$ and is strict, then $B A$ is not Hermitian and the display shows that neither $A \psi(B)$ nor $\psi(B) A$ is Hermitian and $\psi(B)$ is strict. Assume that $\psi(B)$ is not strict. Then, by Lemma $6, \psi(B) \epsilon C_{4}(A)$. But we have shown that $\psi$ sends only elements of $C_{3^{\prime}}(A)$ to $C_{4}(A)$ and therefore $B$ is not strict. Thus if $B \epsilon C_{2}(A)$ and is strict, $\psi(B) \epsilon C_{2}(A)$ and is strict.

We observe that if $A$ is $E P r$, then by the same kind of proof given for Theorem $3, \psi_{1}(B)=B^{*} A^{*} B$ is in $C_{2}(A)$ and is $E P r$ whenever $B \epsilon C_{2}(A)$. Moreover, when $B \epsilon C_{2}(A), \psi_{1}(B)=B$ if and only if $B$ is $E P r$. We then have the following parallel of Theorem 7: $\psi_{1}$ sends all of $C_{3}(A)$ to $C_{4}(A)$ and the remainder of $C_{2}(A)$ into the set of all strict $C_{2}$-inverses of $A$ which are $E P r$.

\section{References}

[1] R. C. Bose, Lecture Notes on Analysis of Variance, University of North Carolina, Chapel Hill, 1959.

[2] C. Eckart and G. Young, A principle axis transformation for non-hermitian matrices, Bull. Am. Math. Soc. 45, 118121 (1939).

[3] A. J. Goldman and M. Zelen, Weak generalized inverses and minimum variance linear unbiased estimation, J. Res. NBS 68B (Math. and Math. Phys.) No. 4, 151-172 (1964).

[4] J. Z. Hearon, Construction of EPr generalized inverses by inversion of nonsingular matrices, J. Res. NBS 71B (Math. and Math. Phys.) Nos. 2 and 3, 57-60 (1967).

[5] J. Z. Hearon, A generalized matrix version of Rennie's inequality, J. Res. NBS 71 B (Math. and Math. Phys.) Nos. 2 and 3, 61-64 (1967).

[6] J. Z. Hearon, Symmetrizable generalized inverses of symmetrizable matrices, J. Res. NBS 71B (Math. and Math. Phys.) No. 4, 229-231 (1967).

[7] J. Z. Hearon and J. W. Evans, Differentiable generalized inverses, J. Res. NBS 72 B (Math. Sci.) No. 2, (1968).

[8] I. J. Katz and M. H. Pearl, On EPr and normal EPr matrices, J. Res. NBS 70B (Math. and Math. Phys.) No. 1, 47-77 (1966).

[9] M. H. Pearl, On generalized inverses of matrices, Proc. Cambridge Philos. Soc. 62, 673-677 (1966).

[10] R. Penrose, A generalized inverse for matrices, Proc. Cambridge Philos. Soc. 51, 406-418 (1955).

[11] C. A. Rohde, Contributions to the theory, computation and application of generalized inverses, Ph.D. Thesis, North Carolina State University, Raleigh, 1964.

[12] C. A. Rohde, Some results on generalized inverses, SIAM Rev. 8, 201-205 (1966).

(Paper 72B2-263) 